\title{
Migraine pain location: how do children differ from adults?
}

\author{
A. Chakravarty $\cdot$ A. Mukherjee $\cdot$ D. Roy
}

Received: 28 May 2008/Accepted: 5 September 2008/Published online: 15 October 2008

(C) Springer-Verlag 2008

\begin{abstract}
The present report provides a detailed head to head comparative study of migraine pain location at onset and during established headache between children and adults belonging to the same ethnic group. Migraine pain location in 200 children and adolescents had already been reported (Chakravarty et al. in Cephalalgia 27:1109-1114, 2007). The present report includes data collected simultaneously and in a similar fashion from 800 adult migraineurs. Significant differences have been noted in migraine pain location. Unilateral onset pain is common in adults, side locked and vertex onset pain unusual in children, holocranial onset pain much common in children and cervicooccipital onset pain much less common in children. There have been differences in evolution of migraine pain as well. During established headache unilateral pain was recorded in only a small proportion of children $(10.5 \%)$ whereas it was noted in many adults' subjects $(40.5 \%)$. Such a detailed comparative study had not been made earlier.
\end{abstract}

Keywords Migraine pain location - Pediatric migraine · Adult migraine

\section{Introduction}

The 'footnote' in the International Classification of Headache Disorders, second edition (ICHD-2) [1] for migraine

A. Chakravarty $\cdot$ A. Mukherjee $\cdot$ D. Roy

Department of Neurology, Vivekananda Institute of Medical

Science, Calcutta, India

A. Chakravarty ( $\square)$

1E 1202 Avishikta 11, Calcutta 700078, India

e-mail: saschakra@yahoo.com without aura (1.1) recognized some differences between pediatric and adult migraine. It only briefly mentioned the differentiating feature in migraine pain location: 'migraine headache is commonly bilateral in young children; an adult pattern of unilateral pain usually emerges in late adolescence or early adult life' and 'migraine headache is usually fronto-temporal'. ICHD-2 also comments on the rarity of occipital headache in children and calls for diagnostic caution to exclude structural lesions; however, no study had ever made a head to head comparison between migraine pain location both at onset and during established headache between children and adults. In fact literature on migraine pain location remains on the whole sparse. Comments related to location of migraine headache in children have been made by Wober-Bingol et al. [2], Winner et al. [3], Lewis and Winner [4] and Hershey et al. [5]. A detailed pain location study in adults has recently been reported by Kelman [6]. More recently, the present authors reported their experience on migraine pain location at onset and during established headaches in a group of 200 children living in the eastern Indian state of West Bengal [7]. In the present report the data from the aforementioned study would be compared with those obtained from a similar study in relation to migraine pain location at onset and during established headaches in 800 adult patients which was carried out simultaneously with the study in children.

\section{Materials and methods}

The reported studies were carried out between September 2003 and December 2005 at the neurology out-patient department of a large general teaching hospital (550 beds) in the city of Calcutta in the eastern part of India. In the pediatric study, a total of 262 subjects (age 7-15 years) 
diagnosed with migraine (ICHD-2 criteria-location excluded) and having at least two attacks of acute migraine per month during the preceding 6 months of recruitment and who were not on any form of prophylactic medication were assessed through a semistructured questionnaire. Patients with chronic migraine were not included in either the children or adult study. The methodology had been detailed earlier [7]. The first consecutive 200 subjects giving consistent (or near consistent) responses to questions relating to pain location, both at onset and during established phase of headaches, were included for analysis.

The adult study was carried out simultaneously and in a similar fashion. A total of 1,172 adult patients $(>15$ years of age) with migraine (ICHD-2 criteria- location excluded) were originally recruited and studied with a similar headache questionnaire in three stages as described in case of children [7].

At first visit, each patient was evaluated by one of the three participating consultants (authors) to confirm diagnosis of migraine, identification of subtype and arranging of relevant investigations to exclude secondary headaches as and when needed (imaging done in 162 patients and positive in only two patients).

During the second visit, 1 week (on average) later, each patient was evaluated through a semi-structured questionnaire by a trainee physician specifically assessing the site of location of onset pain and its subsequent location during established attacks in the majority $(>50 \%)$ of headache spells in the preceding 6 months. Patients were asked to put their hands at the sites of onset pain, show with their hands the subsequent spread of the pain (if possible) and finally the location of the pain during the established phase of the headache. Description of terms used for anatomical sites of location:

\begin{tabular}{|c|c|c|}
\hline & Ocular & Eye/orbit \\
\hline & Frontal & Unilateral—one side of forehead \\
\hline & & Bifrontal—whole of forehead \\
\hline 3. & Temporal & Unilateral/bilateral temple region \\
\hline 4. & Hemicranial & $\begin{array}{l}\text { Unilateral temporal and parietal regions; may } \\
\text { involve ipsilateral frontal/orbital regions and } \\
\text { occasionally ipsilateral occipital area }\end{array}$ \\
\hline & $\begin{array}{l}\text { Vertex/ } \\
\text { Central }\end{array}$ & Center of cranium \\
\hline 6. & Occipital & Back of head above hairline-generally both sided \\
\hline 7. & Cervical & Back of neck below hairline—generally both sided \\
\hline & Holocranial & $\begin{array}{l}\text { Whole forehead, both temporal and parietal as well } \\
\text { as central cranial area and occipital region }\end{array}$ \\
\hline
\end{tabular}

It may be noted that, while noting the location of pain, especially pain at onset, the area mostly affected was taken into account. For example, pain involving one side of forehead but extending a little into the temple region, had been designated as frontal onset and similarly vice versa. Indeed, this may be somewhat artificial but this had been necessary for making the final analysis a little simplified but still meaningful.

During a third visit, usually a month later, each patient was asked the same question relating to the location of pain by one of the authors (consultants) and compared to the responses in the interview by the trainees. In subjects in whom some discrepancies had been noted between the two responses the patients were offered the first responses and given the choice.

Patients $(n=102)$ giving grossly discrepant and vague responses (not really of localizing value) were excluded from the study. Also excluded were patients who failed to turn up for the second or third stage of interview even after reminder $(n=88)$. All response sheets were carefully evaluated by the corresponding author and only the first 800 subjects giving consistent (or near consistent) responses to questions relating to pain localization both at onset and during established phase of headache were included for analysis. The data obtained from these two studies were subsequently compared.

\section{Results}

Table 1 is a composite table showing migraine pain location at onset and during established headaches (vis-à-vis onset location) in children $(n=200)$ and adults $(n=800)$ in $>50 \%$ of headache spells.

Observations made in the pediatric study of 200 patients had already been detailed [7].

To summarize:

1. Migraine in children and adolescents in the study appears to be a slightly male-dominant disease. This might have been contributed by referrel pattern.

2. Migraine with aura (MA) is rare in Indian patients and migraine in children and adolescents is no exception.

3. Only $20.5 \%$ of children had unilateral onset of pain during acute migraine attacks. Of these $(n=41)$, most $(60.9 \%)$ had unilateral frontal location; ocular pain occurred in $26.8 \%$ and temple pain in only $12.2 \%$. In no subjects had onset pain been side locked.

4. Pain was bilateral in $33 \%$ of subjects $(n=66)$. Bifrontal pain at onset was found to be most common (56.1\%) and was less commonly found in both eyes (27.3\%) and bitemporally (16.6\%). None had onset pain localizing in the vertex region. 
Table 1 Location of migraine pain at onset and subsequent evolution during established adults. (in $>50 \%$ occasions) headaches in children and

\section{Location of onset pain}

Pain location during established headaches

A. Unilateral
1. Ocular

2. Frontal

3. Temporal

Side locked

B. Bilateral/central

1. Bilateral ocular

\section{a. Remained same \\ b. Holocranial}

2. Bifrontal

a. Remained same

b. Holocranial

3. Bitemporal

4. Vertex
a. Remained same
b. Holocranial

a. Remained same

b. Hemicranial

c. Bilateral/holocranial

C. Occipito-cervical

* Important differences between children and adults. Chi square test applied

$\begin{array}{lll} & 23(11.5 \%)^{*} & 211(26.38 \%)^{*} P<0.001 \\ \text { a. Remained same } & 6(26.1 \%) & 75(35.55 \%)^{*} \\ \text { b. Hemicranial } & \text { Nil* } & 49(23.22 \%)^{*} \\ \text { c. Bilateral/holocranial } & 17(73.9 \%) & 87(41.23 \%) \\ & 70(35 \%)^{*} & \text { Nil* } P<0.001\end{array}$

Nil* $P<0.001$
Children $(n=200) \quad$ Adults $(n=800)$

$41(20.5 \%)^{*}$

$11(26.8 \%)$

$6(54.5 \%)$

$1(9.1 \%)^{*}$

$4(36.4 \%)^{*}$

$25(60.98 \%)$

$7(28.0 \%)$

$4(16.0 \%)$

$14(56.0 \%)$

$5(12.2 \%)$

Nil

$3(60 \%)$

$2(40 \%)$

None*

$66(33 \%)$

$18(27.3 \%)$

$11(61.1 \%)$

$7(38.9 \%)$

$37(56.1 \%)$

$8(72.7 \%)$

$3(27.3 \%)$

$11(16.6 \%)$

$8(72.7 \%)$

$3(27.3 \%)$

Nil*

$331(41.38 \%) * P<0.001$

$176(53.17 \%)$

$81(46 \%)$

$65(36.9 \%) * P<0.001$

$30(17.0 \%) * P<0.001$

$27(8.16 \%)$

$8(29.6 \%)$

$4(14.8 \%)$

$15(55.5 \%)$

$128(38.67 \%)$

$68(53.1 \%)$

$40(31.3 \%)$

$20(15.6 \%)$

$105(31.72 \%) * P<0.001$

$258(32.25 \%)$

$36(13.96 \%)$

$25(69.4 \%)$

$11(30.6 \%)$

$18(6.97 \%)$

$8(44.4 \%)$

$10(55.5 \%)$

$132(51.16 \%)$

$92(69.7 \%)$

$40(30.3 \%)$

$72(27.91 \%) * P<0.001$
5. Holocranial location of pain at onset was common, occurring in $35 \%(n=70)$ of subjects.

6. Onset pain localizing at the occipito-cervical region was found in $11.5 \%(n=23)$ of subjects, and was mostly bilateral.

7. In $31.7 \%$ of subjects with unilateral ocular, frontal and temporal location of pain at onset, pain remained at the same site during established headaches.

8. Only $19.5 \%(n=8)$ of subjects with unilateral ocular, frontal and temporal location of pain at onset subsequently developed ipsilateral hemicranial headache.

9. In contrast $48.8 \%$ of subjects with unilateral location of pain at onset, subsequently developed bilateral or holocranial headaches.
10. In $42.4 \%$ of subjects with bilateral (both eyes, bifrontal and bitemporal) location of pain at onset, pain remained at the same sites during established headache spells, but in the majority $(57.8 \%)$, during established attacks pain had been holocranial.

11. All subjects with holocranial location of pain at onset $(n=70)$ continued to experience pain at the same location, but of varying intensity.

12. Of the small number of subjects with occipitocervical location of pain at onset $(n=23)$, most $(n=17 ; 73.9 \%)$ subsequently had holocranial pain and in $26.1 \%(n=6)$ pain remained localized at the same site. None developed hemicranial headaches. 
13. In all, during the established migraine headache phases, only $10.5 \% \quad(n=21)$ of all subjects ( $n=200)$ had experienced strictly unilateral pain. The vast majority experienced bilateral or holocranial headaches $(n=173,86.5 \%)$ during established migraine. A minority of subjects $(n=6,3 \%)$ experienced only occipito-cervical pain during established migraine attacks.

The results in the adult study (as depicted in Table 1) may be summarized as follows:

1. Demographic features include: $N=800 ; \mathrm{M}: \mathrm{F} 1: 4.56$; age 16-42 years (mean 26 years); duration 118 years(mean 6.8 years); migraine without aura $668(83.5 \%)$; migraine with typical aura 18 $(2.25 \%)$; probable migraine $114(14.25 \%)$. Migraine with aura appears to be uncommon in Indian subjects in the present study.

2. Exactly $41.38 \%$ of subjects had unilateral onset of migraine headache of which little over one-half $(53.17 \%)$ had ocular location of pain at onset and little over one-third $(38.67 \%)$ had location of pain at onset in the temple region. In about one-third of subjects with unilateral onset, pain had been side locked.

3. Bilateral/central (vertex) location of pain at onset was experienced by $32.25 \%$ of subjects. Majority $(51.16 \%)$ of them had bitemporal pulsating pain to start with followed by $27.91 \%$ experiencing onset pain at the vertex region.

4. Exactly $26.38 \%$ of subjects noted a cervico-occipital location of pain at onset, of which $14.63 \%$ had predominantly occipital region onset and $11.75 \%$ had predominantly cervical region onset of pain. As in many cases, it had been difficult to clearly distinguish between cervical or occipital location of onset pain; these two areas had been grouped together

5. In $47.4 \%$ of subjects with unilateral ocular, frontal and temporal location of pain at onset, pain remained at the same site during established headaches.

6. Approximately one-third of subjects with location of pain at onset in unilateral ocular, frontal and temporal regions subsequently developed ipsilateral hemicranial pain during established spells of migraine headaches.

7. Only $19.6 \%$ of subjects with unilateral location of pain at onset developed bilateral or holocranial headaches during established phase of the migraine attack.

8. In $56.98 \%$ of subjects with bilateral or vertex location of pain at onset $(n=258)$, pain remained at the same site during established migraine attacks. About $70 \%$ of subjects with bitemporal onset of throbbing pain, continued to experience pain at the same site but in about $30 \%$ pain subsequently became holocranial. Over half $(56.9 \%)$ of subjects having onset pain at vertex region, experienced holocranial headaches subsequently.

9. In $41.23 \%$ of subjects with cervico-occipital location of pain at onset subsequently experienced holocranial headaches. About a quarter of subjects subsequently developed hemicranial headaches.

10. In all, although during established migraine attacks $40.5 \%$ of subjects experienced strictly unilateral pain, only $20.88 \%$ had truly hemicranial headaches.

\section{Discussion}

On the whole literature documenting migraine pain location both in adults and children is sparse. In children, Wober-Bingol et al. noted unilateral pain in $29.8 \%$ and bilateral pain in $55.4 \%$ of their subjects. In a recent review, Lewis and Winner mentioned unilateral location as uncommon in childhood migraine wherein a bifrontal or retro-orbital localization occurred more frequently. More recently, Hershey et al. commented that one of the most common reasons for children not meeting the ICHD-2 criteria fully is the requirement of unilateral location. In adults, Kelman reported that highest location frequencies were in the eyes, temporal and frontal regions. The lowest were diffusely over vertex. The intermediate were in the occipital and neck areas. All these studies reported migraine pain location during established headaches and unlike the present report did not highlight on pain location at onset and its subsequent evolution.

The purpose of the present report had been, however, to document differences in migraine pain location at onset and during established headache between children and adult subjects. Significant differences have been noted and indicated in Table 1. The differences observed may be little elaborated as follows:

1. Unilateral onset pain is twice as common in adults than children. In children commonest unilateral location site in frontal $(60.9 \%)$ whereas it is ocular in adults $(53.17 \%)$ followed by temporal $(38.67 \%)$.

2. Side locked onset pain is unusual in children.

3. Vertex location of onset pain is unusual in children.

4. Holocranial onset pain occurs in over one-third of children but unusual in adults.

5. Cervico-occipital onset pain does occur in small proportion $(11.5 \%)$ of children but much less common than in adults $(26.43 \%)$. In children most became holocranial later but in adults many experienced hemicranial headaches. 
6. Transformation to hemicranial pain from unilateral ocular, frontal or temporal pain occurred more frequently in adults than children (33 vs. 19.5\%). Majority of children with unilateral onset pain developed bilateral or holocranial pain $(48.5 \%)$ in contrast to adults $(19.6 \%)$.

7. Unilateral pain during established headache occurs in only small proportion of children $(10.5 \%)$ but occurs much more commonly in adults $(40.5 \%)$.

Although brief mention about difference in pain location between children and adults had been mentioned in the ICHD-2, [1] no detailed comparative study like the present report had been made earlier. The present study clearly highlights that adult migraine in contrast to pediatric, is mostly an unilateral phenomenon at onset or during established headaches. The study clearly points out the need for a separate diagnostic criterion for migraine in children stressing the inclusion of bilateral and holocranial location of pain.

The exact cause for this phenomenological difference between adults and children is not known. It can only be postulated that this may be the result of differences in degree of brain maturation comprising myelination, new synapse formation and synaptic reorganization. Clearly, further studies are warranted specially localization studies in an age stratified manner to more clearly highlight the evolution of a mostly bilateral phenomenon into a mostly unilateral phenomenon with advancing age from childhood to adult life.

Conflict of interest None.

\section{References}

1. Headache Classification Subcommittee of the International Headache Society (2004) The International Classification of Headache Disorders, 2nd edn. Cephalalgia 24(Suppl 1):8-160

2. Wober-Bingol C, Wober C, Karwautz A, Vesely C, WagnerEnnsgraber C, Amminger GP et al (1995) Diagnosis of headache in childhood and adolescence: a study in 437 patients. Cephalalgia 15:13-21

3. Winner P, Martinez W, Mate L, Bello L (1995) Classification of paediatric migraine: proposed revisions to the IHS criteria. Headache 35:407-410

4. Lewis DW, Winner P (2001) Migraine, migraine variants and other primary headache syndromes. In: Winner $\mathrm{P}$, Rothner $\mathrm{AD}$ (eds) Headache in children and adolescents. B C Decker Inc., Hamilton, pp 60-86

5. Hershey AD, Winner P, Kabbouche MA, Cladstein J, Yonker M, Lewis D et al (2005) Use of the ICHD-II criteria in the diagnosis of pediatric migraine. Headache 45:1288-1297

6. Kelman L (2005) Migraine pain location: a tertiary care study of 1283 migraineurs. Headache 45:1038-1047

7. Chakravarty A, Mukherjee A, Roy D (2007) Migraine pain location at onset and during established headaches in children and adolescents: a clinic based study from eastern; India. Cephalalgia 27:1109-1114 